\title{
Passive sampling: partition coefficients for a silicone rubber reference phase.
}

\author{
YATES, K., DAVIES, I., WEBSTER, L., POLLARD, P., LAWTON, L. and \\ MOFFAT, C.
}




\section{Passive sampling: Partition coefficients for a silicone rubber reference phase}

2 Kyari Yates ${ }^{1,2}$, Ian Davies ${ }^{1}$, Lynda Webster ${ }^{1}$, Pat Pollard ${ }^{2}$, Linda Lawton ${ }^{2}$ and Colin

3 Moffat $^{1,2}$

41 Fisheries Research Services Marine Laboratory, 375 Victoria Road, Aberdeen, AB11 9DB, UK

52 School of Life Sciences, Robert Gordon University, Aberdeen, UK

\section{Abstract}

10 Silicone rubber sheeting can be used as a passive sampling device for hydrophobic

11 organic contaminants in the environment to determine the available concentrations in

12 water and sediments. Reliable sampler-water partition coefficients are required to

13 determine the sampling rates and the dissolved contaminant concentrations in water and

14 in sediment pore water. $\log$ partition coefficients $\left(\log K_{s r, w}\right)$ for silicone rubber-water

15 have been estimated for 32 polycyclic aromatic hydrocarbons (PAHs), 2 deuterated PAH

16 analogues and 32 chlorobiphenyls (CBs) using the cosolvent method, with methanol as

17 cosolvent. Strong linear relationships were found with literature values for the

18 corresponding $\log$ octanol-water partition coefficients $\left(\log K_{\text {ow }}\right)$ for both CBs and PAHs,

19 confirming that partitioning into the silicone rubber is strongly determined by the

20 hydrophobicity of the compounds, which suggests $\log K_{\text {ow }}$ is a good predictor of

$21 \log K_{s r, w}$ and that absorption is the main mechanism for accumulation of analytes into

22 the silicone rubber polymer. 


\section{Introduction}

3 The determination of the dissolved concentrations of priority hydrophobic organic

4 contaminants (HOCs) such as polycyclic aromatic hydrocarbons (PAHs) and

5 chlorobiphenyls (CBs) is a difficult task due to their low concentrations and the problem

6 of incomplete phase separation between particle-bound and dissolved analytes ${ }^{1}$. The

7 problems are even more pronounced when the determination is required in sediment pore

8 waters, as large volumes of pore water are required. The development of passive

9 sampling methods, using solid or liquid reference phases, allows direct measurement of

10 exposure to dissolved hydrophobic organic contaminants. Single phase sampling devices,

11 such as low density polyethylene (LDPE strips) ${ }^{1}$, polyoxymethylene strips (POM) ${ }^{3}$,

12 Tenax $^{4}$, polydimethylsiloxane (PDMS) ${ }^{5}$, and silicone rubber ${ }^{2,6,7}$ have been developed

13 in addition to the two-phase semi-permeable membrane devices (SPMDs) ${ }^{8,9}$ developed

14 earlier to determine the free dissolved HOC concentrations. They are easy to construct,

15 re-usable and of low cost ${ }^{6}$. Rusina et al. ${ }^{6}$ discussed the properties of materials for

16 passive samplers and proposed that silicone rubbers can be attractive reference phases

17 due to their high partition coefficients and low transport resistances.

19 Using reference (sampling) phases, such as silicone rubber, that equilibrate with the

20 surrounding medium, the partition coefficient can be used, together with the

21 concentration in the sampler, to determine the freely dissolved concentration in the

22 environmental medium ${ }^{10}$, to estimate the sampling rates of added performance reference

23 compounds ${ }^{11}$ that have dissipated from the passive sampling device and subsequently the

24 equilibration rate constants which are used to determine the sampling scenario (kinetic or

25 equilibrium), as not all compounds would have attained equilibrium. Consequently,

26 passive sampling using a reference phase that equilibrates with the dissolved

27 concentration in the sampling medium is attractive. The ratio of the concentration in the

28 sampler to that in the surrounding water at equilibrium yields the sampler-water partition

29 coefficient as described in equation 1 :

$30 \quad K_{s, w}=\left(\frac{C_{s}}{C_{w}}\right)$ 
1 where $K_{s, w}$ is the sampler-water partition coefficient, $C_{S}$ and $C_{w}$ is the concentration in

2 the sampler and water respectively. The sampling rate is calculated from equation $2^{1}$ :

$3 \quad R_{s}=k_{e} K_{s, w} M_{s}$

4 where $R_{s}$ is the sampling rate, $k_{e}$ is the exchange coefficient (assuming first order kinetics

5 during sampling) and $M_{s}$ is the sampler mass. The $k_{e}$ can be estimated from the release of

6 performance reference compounds, spiked onto the sampler using

$7 \quad k_{e}=-\frac{\ln \left(N^{t} / N^{0}\right)}{t}$

$8 N^{t}=$ Amount of PRC left in sampler at the end of exposure time $t, N^{0}=$ Amount of

9 PRC spiked onto the sampler at the start of the exposure

11 It is therefore necessary to determine the partition coefficients that accurately describe

12 partitioning in multi-compartment systems ${ }^{5,10-13}$, particularly those between the

13 reference sampling phase (e.g. silicone rubber) and water. The dissolution of PAHs and

14 CBs in water is quite difficult due to their hydrophobic nature, and as a consequence may

15 adhere to glass surfaces used in the experimental set up and yield variable water

16 concentrations, which complicates the determination of partition coefficients. The

17 partitioning of HOCs between environmental media is mainly determined by the aqueous

18 solubility of the HOCs which may be modified (increased) by the addition of organic

19 solvents ${ }^{14}$. Therefore, we have measured the silicone rubber-water partition coefficients

20 of a series of HOCs (PAHs and CBs) with $\log K_{\text {ow }}$ values for the compounds studied

21 ranging from 3.3 to 8.2 using the cosolvent method, with methanol as cosolvent. This was

22 carried out by determining log partition coefficients at different co-solvent-water volume

23 percentages and extrapolating the $>20 \% \mathrm{v} / \mathrm{v}$ methanol portion of the linear curve of log

24 partition coefficient versus percent methanol to zero percent methanol which yields the

25 true partition coefficient of the HOC between silicone rubber and water ${ }^{15-17}$. The

26 estimation of partition coefficients over a wide range of methanol percentages reduces

27 some of the errors normally associated with measurements of partition coefficients. 


\section{Materials and methods}

3 Materials

5 AlteSil ${ }^{\text {TM }}$ Silicone rubber sheet manufactured from translucent, food grade silicone

6 rubber, with a hardness of 60 Shore A, $(600 \times 600 \mathrm{~mm}, 0.5 \mathrm{~mm}$ thick $)$ were purchased

7 from Altec Products, Ltd, Cornwall, UK. HPLC grade solvents (ethyl acetate, acetone,

8 methanol and 2-methylpentane) were purchased from Rathburn Chemicals Ltd, Scotland,

9 UK. Certified solid standards for PAHs (including deuterated PAHs) and CBs were

10 obtained from QMX Laboratories, Essex, UK and dissolved in 2-methylpentane (PAHs)

11 and 2, 2, 4-trimethylpentane (CBs) to obtain required concentrations of spiking solutions.

12 The silicone rubber sheets were cut into $6 \times 4 \mathrm{~cm}$ pieces and pre-extracted with ethyl

13 acetate in a Soxhlet apparatus for 4 days to remove any low molecular weight oligomers

14 or residues that may interfere with subsequent analyses. Milli-Q water (18.2 M $2 . \mathrm{cm})$ was

15 used throughout.

\section{Loading of Compounds on silicone rubber}

19 Test compounds (PAHs and CBs) were loaded onto the silicone rubber sheets using the

20 spiking method described by Booij et al. ${ }^{2}$. Briefly, $100 \mathrm{ml}$ of methanol in an amber glass

21 jar was spiked with known concentrations of the PAHs and CBs of interest (Table 1) and

22 silicone rubber sheets added. The glass jar was shaken for $2 \mathrm{~h}$ on an orbital shaker at 200

$23 \mathrm{rpm}$ followed by addition of water to obtain $80 \% \mathrm{v} / \mathrm{v}$ methanol solution and further

24 shaken for $6 \mathrm{~h}$ with a subsequent addition of water to obtain $50 \% \mathrm{v} / \mathrm{v}$ methanol solution.

25 This was followed by a final shaking overnight at room temperature. Reproducibility of

26 the spiking method was always better than $5 \%$ within each batch of spiked silicone

27 rubber. 


\section{Partition coefficient determination}

3 The co-solvent method ${ }^{15,16}$ was used with methanol as co-solvent. The silicone rubber

4 sheets, loaded with appropriate amounts of test PAHs and CBs, were introduced into 11

5 glass bottles (one sheet per bottle) containing $900 \mathrm{ml}$ of the water-methanol mixture at a

6 range of 7 fractions $(20-50 \% \mathrm{v} / \mathrm{v}$ methanol). An un-spiked sheet was added to a bottle

7 containing $900 \mathrm{ml}$ of $20 \% \mathrm{v} / \mathrm{v}$ methanol solution as procedural blank. Duplicate bottles

8 were then placed on an orbital shaker at $150 \mathrm{rpm}$ for 15 days ${ }^{17,18}$ (Smedes ${ }^{17}$ used 20

9 days in his work) during which time equilibrium can be assumed to have been attained.

10 Other studies have shown organic compounds, e.g. CB29, D10-phenanthrene, phenol,

11 benzene, dichlorobenzene, etc to equilibrate in silicone membranes within hours ${ }^{1,19}$.

12 Sorption of analytes to the glass container is considered negligible ${ }^{16,17}$ and the

13 concentrations in the water phase were generally measurable. The bottle caps were lined

14 with solvent-washed aluminium foil to prevent sorption onto the plastic caps. The

15 silicone rubber sheets were removed from the bottles and gently wiped dry with paper

16 rolls before extraction.

18 Deuterated PAH internal standards (D8-naphthalene, $\mathrm{D}_{10}$-biphenyl, $\mathrm{D}_{10}$-anthracene, $\mathrm{D}_{8}$ -

19 dibenzothiophene, $\mathrm{D}_{10}$-pyrene and $\mathrm{D}_{12}$-benzo $[a]$ pyrene) were added to the methanol-

20 water mixtures in the 11 bottles and transferred into separation funnels. These were

21 liquid-liquid extracted in separating funnels using $2 \times 60 \mathrm{ml}$ dichloromethane and the

22 extracts dried over anhydrous sodium sulphate. The extracts were rotary evaporated to

$232 \mathrm{ml}$ and $2 \times 25 \mathrm{ml}$ 2-methylpentane added followed by concentration, first by rotary

24 evaporation, then by nitrogen blow down to $1 \mathrm{ml}$ and split into 2 parts: 1 fraction was

25 analysed by gas chromatography-mass selective detection (GC-MS) for PAHs and the

26 other by gas chromatography-electron capture detection (GC-ECD) for CBs. The silicon

27 rubber sheets were Soxhlet extracted using a 2-methylpentane: acetone $(3: 1 \mathrm{v} / \mathrm{v})$ mixture

28 for $6 \mathrm{~h}$ and the same suite of deuterated PAH internal standards added, then exchanged

29 into 2-methylpentane by the addition of $2 \times 25 \mathrm{ml}$ of 2 -methylpentane. The extract was

30 concentrated by rotary evaporation followed by nitrogen blow down to $1 \mathrm{ml}$ which was

31 then split into 2 fractions by weight for PAHs and CBs. An aliquot of the PAH fraction 
1 was fractionated by isocratic, normal phase high performance liquid chromatography

2 using a Genesis silica column and eluted with 2-methylpentane into aliphatic and

3 aromatic fractions. The aliphatic fraction was discarded and the aromatic fraction

4 collected and concentrated before analysis for PAHs. Similarly an aliquot of the CB

5 fraction was fractionated as above and the eluate from the first 6 min (predetermined

6 using a solution of $\mathrm{CBs}$ ) collected and 2, 4-dichlorobenzyl alkyl ethers internal standard

7 added, then concentrated before analysis for CBs. Recoveries calculated on the basis of

8 the sum of the amounts determined in the methanol-water mixtures and amounts in the

9 sheets relative to the amount loaded onto the sheets were $86 \pm 20 \%$ for the CBs and 101

$10 \pm 16 \%$ for PAHs.

12 Confirmatory data were obtained using silicone rubber sheeting (Vizo, Technirub,

13 Netherlands) for which partition coefficients had been independently determined ${ }^{17}$. Vizo

14 and Altec sheeting were equilibrated together by loading them with the test compounds,

15 in triplicate, in amber glass bottles as previously described. As the two materials were

16 equilibrated in the same methanol-water phase, measurements of the concentrations in the

17 methanol-water phase were not necessary since:

$18 \quad\left(\frac{C_{s r}}{K_{s r, w}}\right)_{1}=\left(\frac{C_{s r}}{K_{s r, w}}\right)_{2}=C_{w}$

19 Implying $\left(K_{s r, w}\right)_{2}=\left(\frac{\left(C_{s r}\right)_{2}}{\left(C_{s r}\right)_{1}}\right) \cdot\left(K_{s r, w}\right)_{1}$

20 where the subscripts sr- denotes silicone rubber, w-water and 1, 2 refer to Vizo, and Altec

21 sheeting respectively.

\section{Chromatographic Analysis}

25 The concentrations and composition of the PAHs were determined by gas

26 chromatography mass selective detection (GC-MS) using an HP6890 Series Gas

27 Chromatograph interfaced with an HP5973 MSD fitted with a cool on-column injector as

28 described by Webster et al. ${ }^{20}$. Briefly, a non-polar HP5 $(30 \mathrm{~m} \times 0.25 \mathrm{~mm}$ id, $0.25 \mu \mathrm{m}$

29 film thickness; Agilent Technologies, Stockport, England) column was used for the 
1 analyses with helium as the carrier gas, controlled using the constant flow mode at $0.7 \mathrm{ml}$

$2 \mathrm{~min}^{-1}$. Injections were made at $50^{\circ} \mathrm{C}$ and the oven temperature held constant for $3 \mathrm{~min}$.

3 Thereafter, the temperature was raised at $20{ }^{\circ} \mathrm{C} \mathrm{min}^{-1}$ up to $100{ }^{\circ} \mathrm{C}$, followed by a slower

4 ramp of $4{ }^{\circ} \mathrm{C} \mathrm{min}-1$ up to a final temperature of $270{ }^{\circ} \mathrm{C}$. The MSD was set for selective ion

5 monitoring (SIM) with a dwell time of $50 \mathrm{~ms}$. A total of 30 ions plus the six internal

6 standard ions were measured over the analysis period, thus incorporating 2- to 6- ring,

7 parent and branched PAHs.

9 The concentration and composition of $\mathrm{CB}$ congeners were determined by gas

10 chromatography with electron capture detection (GC-ECD) using a Perkin Elmer Gas

11 Chromatograph Clarus 500 auto system (Perkin Elmer, Beaconsfield, UK) fitted with a

12 cool on-column injector. A non-polar HP 5 column $(60 \mathrm{~m} \times 0.25 \mathrm{~mm}$ id, $0.25 \mu \mathrm{m}$ film

13 thickness; Agilent Technologies, Stockport, England) was used for the analyses along

14 with an uncoated pre-column $(2.5 \mathrm{~m} \times 0.53 \mathrm{~mm}$ id $)$. The carrier gas was hydrogen (1-3

$\left.15 \mathrm{ml} \mathrm{min}{ }^{-1}\right)$ and make-up gas was nitrogen $\left(30 \pm 5 \mathrm{ml} \mathrm{min}^{-1}\right)$. The initial oven temperature

16 was $80^{\circ} \mathrm{C}$ and held for $1 \mathrm{~min}$ after which it was ramped at $3{ }^{\circ} \mathrm{C} \mathrm{min}{ }^{-1}$ to a final

17 temperature of $280^{\circ} \mathrm{C}$ and held at this temperature for $12 \mathrm{~min}$. The chromatograph was

18 calibrated using a series of external standards and the two 2, 4-dichlorobenzyl alkyl

19 ethers. The data were quantified using a Client Server Turbochrom data system (Perkin

20 Elmer, Beaconsfield, UK).

\section{Octanol-water partition coefficients}

24 Octanol-water partition coefficients were obtained from literature. Sangster ${ }^{21}$ compiled

$25 \log K_{o w}$ values reviewed from available literature and gave recommended values for some

26 compounds which were adopted in this study. For compounds where no recommended

27 value was given, average value of the $\log K_{\text {ow }}$ data presented was chosen and where only

28 one value was given this was adopted. 


\section{Results}

\section{Co-solvent method}

5 Silicone rubber-water partition coefficients were calculated (Eq. 1) at each methanol

6 percentage and log linear curves plotted for $K_{s r, w}$ as a function of the methanol volume

7 percent (Figure 1). The partition coefficients by the co-solvent method were estimated

8 from the intercept of the regression lines at $100 \%$ water ( $0 \%$ methanol) thus eliminating

9 the need to measure the partition coefficients directly in pure water. Addition of the

10 cosolvent increases the hydrophobicity of the resulting solvent (water/co-solvent

11 mixture), which increases solubility of the target compounds in the solvent mixture ${ }^{14,16 \text {, }}$

$12 \quad 22,23$. The solubility increases exponentially while the logarithm of partition coefficients

13 will decrease linearly with increasing mole fraction of methanol. Linear regressions were

14 good descriptors of the relationship between $\log K_{s r, w}$ and percent methanol with the

15 intercept representing $\log K_{s r, w}$ at $0 \%$ methanol. $\log K_{s r, w}$ values, along with the $95 \%$

16 confidence interval of the intercept of $\log K_{s r, w}$ - methanol volume percentage are shown

17 in Table 1.

19 For the more hydrophobic compounds, the number of data points used in the regression

20 analysis is often less than 7 due to problems in the accurate measurement of

21 concentrations of HOCs at low percentages of methanol in water. Inclusion of some of

22 these data of relatively high uncertainty would not have improved the quality of the

23 regression analysis or the estimates of partition coefficients. At least five partition

24 coefficients are recommended for the regression, because if fewer partition coefficients

25 are used for extrapolation, the statistical errors in the estimate of the intercept $\left(\log K_{s r, w}\right)$

26 will tend to be large ${ }^{15}$. 
1 Batch incubation of reference phases

3 The cosolvent method can however be laborious and so an attempt was made to estimate

4 the $\log K_{s r, w}$ from a material of known partition coefficients. Therefore using data

5 obtained from the cosolvent method for Vizo sheets ${ }^{17}, \log K_{s r, w}$ values were predicted for

6 the Altec sheets using Eq. 5. Results obtained by incubating the Vizo and Altec silicone

7 rubbers are presented in Table 2 for those compounds for which partition coefficients for

8 Vizo rubber were available.

\section{Discussion}

12 In the absence of direct measurements, estimates of partition coefficients for passive 13 samplers are often obtained from $\log K_{o w}$ values. The measured Altec silicone rubber-

14 water partition coefficients were plotted against corresponding $\log K_{o w}$ values and good

15 linear relationships were found (Figure $2 \mathrm{a} \& \mathrm{~b}$, Table 4). This confirms that partitioning

16 into the silicone rubber is strongly determined by compound hydrophobicity, as has been

17 reported for other materials, such as POM $^{3}$ and PDMS fibres ${ }^{5}$. No significant

18 differences were found (F-test; $\mathrm{p}>0.05$ ) between the $\log K_{s r, w}-\log K_{o w}$ relations

19 depending on the source of the $\log K_{o w}$ data for either the PAHs or CBs. The regressions

20 using different sources of $\log K_{o w}$ differ in their intercepts for PAHs while the slope did

21 not differ significantly ( slope $=0.97 \pm 0.11$; slope $=1.13 \pm 0.19$, Table 4$)$ when the

22 intercepts are ignored. Deviations from linearity could arise from some uncertainty in

23 available $K_{o w}$ values ${ }^{13,24}$ and other factors, such as possible transport resistance of

24 silicone rubber to large molecules (large $\mathrm{K}_{\mathrm{ow}} \mathrm{s}$ ), as had been observed for various tissues

25 and membranes ${ }^{25}$.

27 Combining both classes of compounds also yields a linear relationship (Table 4) but the

28 decrease in the quality of the regression indicates systematic differences in the

29 relationship between $\log K_{s r, w}$ and $\log K_{o w}$ for different classes of compound. (c.f. Vrana 
1 et al. ${ }^{29}$ ). The measured partition coefficients were on average, 0.70 units less than the

2 octanol-water partition coefficients for the CBs and 0.18 units for the PAHs. The

3 regressions of $\log K_{P O M}$ of CBs and PAHs with $\log K_{\text {ow }}$ have been reported ${ }^{3}$ not to differ

4 significantly which allow for pooling of both classes of compounds while in our study the

5 regressions differed significantly (F-test; $\mathrm{p}<0.001)$. The observed differences in the

6 regressions may be due to structural differences between PAHs and CBs. Differences

7 have been shown ${ }^{1,15,30}$ between $\log K_{o c}$ (organic carbon normalised partition

8 coefficients) $-\log K_{\text {ow }}$ regression lines for PAHs and CBs which have been attributed to

9 differences in molecular structure between the two classes of compounds. Similarly,

10 caution has been suggested with regards to estimation of bioconcentration factors (BCF)

11 from $\log$ BCF-log $K_{o w}$ correlations (which are essentially partitioning models between

12 biological membranes and water) for different classes of compounds ${ }^{31,32}$ due to

13 uncertainties in the measurement of both BCF's and $\log K_{o w}$ (especially at $\log K_{o w}>6$ ).

15 SPMD-water partition coefficients (reviewed from literature) are available in Huckins et

$16 a$ al. ${ }^{23}$, and the average $\log K_{\text {spmd,w }}-\log K_{o w}$ values from the various data was described by

17 a quadratic fit with deviations from linearity observed from $\log K_{o w}>5$ possibly due to

18 solute-triolein incompatibility. This was not the case in the good linear relationships

19 found in this study over a $\log K_{o w}$ range of $3-8$, suggesting that such complications do

20 not occur with silicone rubber and that equilibrium partitioning between the silicone

21 rubber and aqueous phases is almost entirely a function of the hydrophobicity of the

22 target contaminants. The need to determine partition coefficients for both the lipid and

23 the membrane as is the case for SPMDs is eliminated as only a single partition coefficient

24 is sufficient to describe partitioning in the silicone rubber sampler.

26 In view of the uncertainties inherent in the measurements of partition coefficients by

27 either the co-solvent or batch incubation method, it is suggested that use can be made of

28 the linear regressions between $\log K_{s r, w}$ and $\log K_{o w}$ to obtain estimates of $\log K_{s r, w}$. The

29 standard errors in the experimental data in Table 1 are on average 2.35 (CBs) and 1.69 
1 (PAHs) times the errors from the predicted values (Table 3) from the $\log K_{s r, w}-\log$

$2 K_{\text {ow }}$ regressions.

3

4 The $\log K_{s r, w}$ values obtained by incubation (referred to as estimated $K_{s r, w}$, Table 2)

5 correlated well $\left(\mathrm{r}^{2}=0.93\right.$, PAHs and $\left.0.90, \mathrm{CBs}\right)$ with the cosolvent method values (Table

6 1), however, there was an observed increase in deviation of $\log K_{s r, w}$ between the two

7 methods with increasing $\log K_{o w}$. Uncertainties in the silicone rubber-water partition

8 coefficients for the Vizo rubber will have added to overall uncertainties in the partition

9 coefficients estimated by this method for the Altec rubber. It may be noted that a quick

10 estimate of $\log K_{s r, w}$ values can be obtained by, equilibrating the silicone rubber of known

11 partition coefficient with an unknown rubber, for example to confirm the quality of a new

12 batch of rubber.

\section{Conclusions}

16 The co-solvent method offers a practical way to estimate partition coefficients for passive

17 sampling materials, such as silicone rubber. The estimated partition coefficients showed

18 strong linear relations with published values for $\log K_{o w}$ confirming that partitioning into

19 the silicone rubber is largely a function of compound hydrophobicity, and that absorption

20 into the silicone rubber is the main mechanism governing retention of analytes in the

21 polymer. It is suggested that best estimates of partition coefficients for silicone rubber

22 may be obtained from regressions between $\log K_{o w}$ and $\log K_{s r, w}$. The fitted values can be

23 used to estimate sampling rates of passive samplers and in the determination of free

24 dissolved concentrations of PAHs and CBs in water and sediment pore water. In cases

25 where no silicone rubber-water partition coefficients are available, estimations can be

26 made using the octanol-water partition coefficients, although measured values are always

27 better. It is also possible to attempt to measure $\log K_{s r, w}$ in pure water, with any

28 agreement of the cosolvent values with water-only values greatly enhancing the 
1 reliability of the final values, although there always will be some uncertainty with the 2 individual methods.

3

4

5

6

7

8

11

12

13

14

15

16

17

18

19

20

21

22

23

24

25

26

27

28

29

30

31 


\section{References}

3 1. K. Booij, J. H. Hoedemaker, and J. F. Bakker, Environ. Sci. Technol., 2003, 37, $4 \quad 4213-4220$

6 2. K. Booij, F. Smedes, and E. M. van Werlee, Chemosphere, 2002, 46, 1157-1161

8 3. M. T. O. Jonker, and A. A. Koelmans, Environ. Sci. Technol., 2001, 35, 37423748

4. G. Cornelissen, H. Rigterink, D. E. M. ten Hulscher, B. A. Vrind, and P. C. M. van Noort, Environ. Toxicol. Chem., 2001, 20, 706-711

5. P. Mayer, W.H.J. Vaes, and J.L.M Hermens, Anal. Chem., 2000, 72, 459-464

6. T. P. Rusina, F. Smedes, J. Klanova, K. Booij, and I. Holoubek, Chemosphere, 2007, DOI: 10.1016/j.chemosphere.2007.01.025

7. L. Wennrich, B. Vrana, P. Popp, and W. Lorenz, J. Environ. Monit., 2003, 5, 813-

8. J. N. Huckins, M. W. Tubergen, and G. K. Manuweera, Chemosphere, 1990, 20,

9. J. N. Huckins, G. K. Manuweera, J. D. Petty, D. Mackay, and J. A. Lebo, Environ. Sci. Technol., 1993, 27, 2489-2496

10. P. Mayer, J. Tolls, J. L. P. Hermens, and D. Mackay, Environ. Sci. Technol., 2003, 37, 187A-191A 
1 11. J. N. Huckins, J. D. Petty, and K. Booij, Monitors of Organic Chemicals in the

2 Environment: Semipermeable membrane devices, Springer Science+Business

3 Media, New York, 2006, pp 183- 186

4

5 12. R. Doong, and S. Chang, Anal. Chem., 2000, 72, $3647-3652$

7 13. T. W. Jabusch, and D. L. Swackhamer, Chemosphere, 2005, 60, 1270-1278

9 14. A. Li, A. W. Andren, S. H. Yalkowsky, Environ. Toxicol. Chem., 1996, 15, $2233-$ 2239

15. M. T. O. Jonker, and F. Smedes, Environ. Sci. Technol., 2000, 34, 1620-1626

16. W. J. M. Hegeman, C. H. van Der Weijden, and J. P. Gustav Loch, Environ. Sci.

$$
\text { Technol., 1995, 29, 363-371 }
$$

18. A. Li, and A. W. Andren, Environ. Sci. Technol., 1994, 28, 47-52

19. P. R. Brookes and A. G. Livingston, J. Memb. Sci., 1995, 104, 119-137

20. L. Webster, L. Phillips, M. Russell, E. Dalgarno, and C. Moffat, J. Environ.

17. F. Smedes, In Comprehensive Analytical Chemistry, ed. R. Greenwood, G. Mills, B. Vrana, Elsevier, Oxford, UK, 1st edn., 2007, vol. 48, ch. 22, pp. 407-448

30 22. R. C. Chawla, K. F. Doura, and D. McKay, Proceedings of the 2001 Conference 
2 23. F. C. Spurlock, and J. W. Biggar, Environ. Sci. Technol., 1994, 28, 1003-1009

4 24. W. M. Meylan, and P. H. Howard, Chemosphere, 2005, 61, 640-644

6 25. C. T. Chiou, Environ. Sci. Technol., 1985, 19, 57-62

8 26. US EPA, EPI SUITE ${ }^{\text {TM }}$ (Estimation Programs Interface) [online] http://www.epa.gov/oppt/exposure/docs/episuite.htm Accessed 12 June 2005

11 27. D. W. Hawker, and D. W. Connell, Environ. Sci. Technol., 1988, 22, 382-387

29. B. Vrana, P. Popp, A. Paschke, and G. Schüürmann, Anal. Chem., 2001, 73, 5191-

28. D. Mackay, W. Y. Shiu, and K. C. Ma, Illustrated Handbook of Physical Chemical Properties and Environmental Fate of Organic Chemicals, Lewis Publishers, London, UK, 1992, vol. 1, pp 327-668

22

31. D. Mackay, Environ. Sci. Technol., 1982, 16, 274-278 


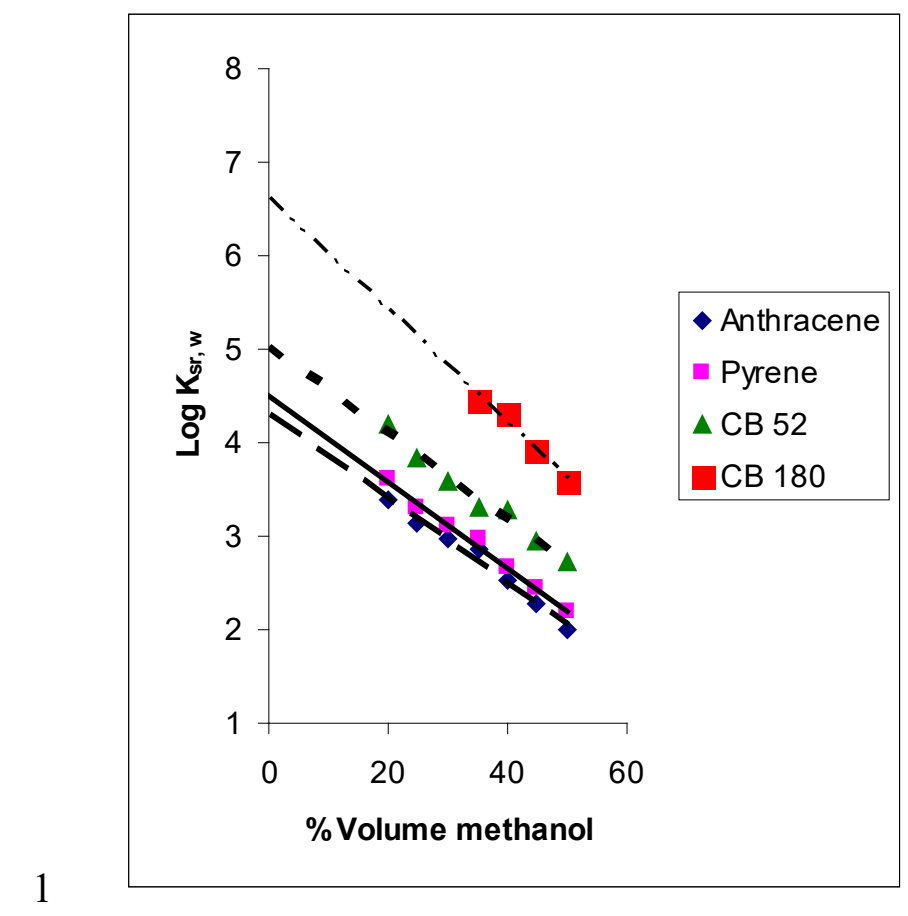

2 Figure 1: Silicone rubber-water partition coefficients as a function of volume percentage

3 methanol for anthracene (diamonds), pyrene (squares), CB 52 (triangle) and CB 180

4 (large squares). Only four points were used for the regression of CB 180, as it was not

5 detected in 20-30 \% volume methanol. Linear regression was used to obtain estimates of 6 intercept. 


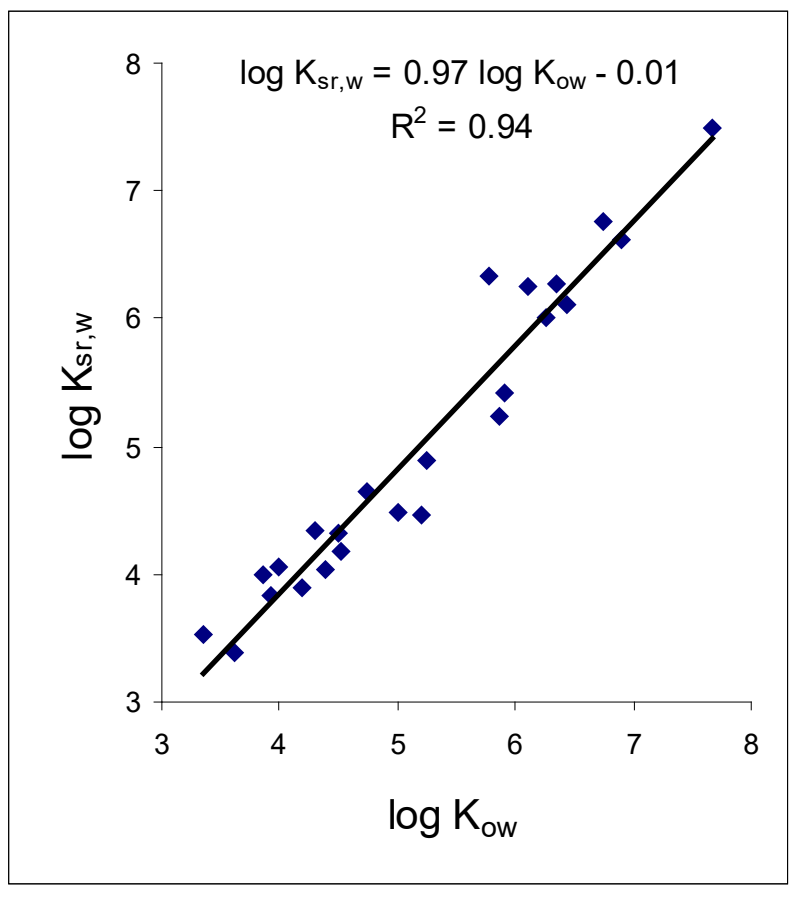

2 Figure 2a: Correlation of $\log K_{s r, w}$ with $\log K_{o w}$ for $24 \mathrm{PAH}$ compounds. $\log K_{s r, w}{ }^{\prime} s$ are 3 mean values of duplicate measurements and $\log \mathrm{K}_{\mathrm{ow}}$ are from Sansgter ${ }^{21}$

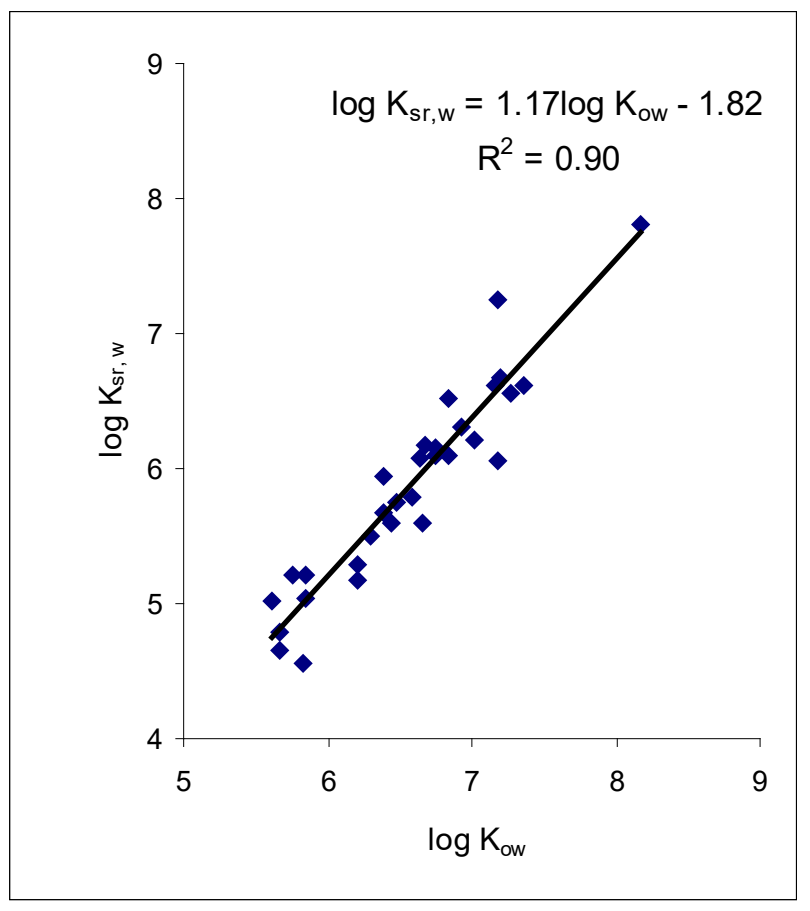

6 Figure 2b: Correlation of $\log K_{s r, w}$ with $\log K_{o w}$ CBs. $\log K_{s r, w}$ are mean values of

7 duplicate measurements and $\log \mathrm{K}_{\mathrm{ow}}$ are from Hawker and Connell ${ }^{27}$ 
1 Table 1: Log Partition coefficients and $95 \%$ confidence interval of estimate of intercept

2 from regression of test compounds

\begin{tabular}{|c|c|c|c|c|c|c|c|c|c|}
\hline Compounds & $\begin{array}{l}\text { Log } \\
\mathrm{K}_{\mathrm{ow}} \\
\end{array}$ & $\begin{array}{c}\log \\
\mathrm{K}_{\mathrm{sr}, \mathrm{W}}\end{array}$ & C.I. ${ }^{1}$ & $\mathrm{n}^{2}$ & Compounds & $\begin{array}{l}\text { Log } \\
\mathrm{K}_{\mathrm{ow}} \\
\end{array}$ & $\begin{array}{c}\log \\
\mathrm{K}_{\mathrm{sr}, \mathrm{W}}\end{array}$ & C.I. ${ }^{1}$ & $\mathrm{n}^{2}$ \\
\hline Naphthalene & 3.35 & 3.53 & 0.40 & 7 & CB 28 & 5.67 & 4.79 & 0.33 & 7 \\
\hline Acenaphthylene & 3.61 & 3.39 & 0.20 & 7 & CB 31 & 5.67 & 4.66 & 0.30 & 7 \\
\hline Acenaphthene & 3.92 & 3.84 & 0.35 & 7 & CB 35 & 5.82 & 4.56 & 0.40 & 7 \\
\hline Fluorene & 4.18 & 3.89 & 0.33 & 7 & CB 44 & 5.75 & 5.21 & 0.32 & 7 \\
\hline 2-Methylnaphthalene & 4.00 & 4.06 & 0.48 & 7 & CB 49 & 5.85 & 5.22 & 0.40 & 7 \\
\hline 1-Methylnaphthalene & 3.87 & 4.00 & 0.50 & 7 & CB 52 & 5.84 & 5.04 & 0.29 & 7 \\
\hline C2-naphthalene & 4.31 & 4.33 & 0.40 & 7 & CB53 & 5.62 & 5.02 & 0.39 & 7 \\
\hline C3-naphthalene & 4.73 & 4.64 & 0.41 & 7 & CB 70 & 6.2 & 5.17 & 0.35 & 7 \\
\hline C4-naphthalene & $5.17^{\mathrm{a}}$ & 5.17 & 0.44 & 7 & CB 74 & 6.2 & 5.29 & 0.34 & 7 \\
\hline Phenanthrene & 4.52 & 4.18 & 0.31 & 7 & CB 97 & 6.29 & 5.49 & 0.32 & 7 \\
\hline Anth & 4.50 & 4.31 & 0.28 & 7 & CB 99 & 6.39 & 5.68 & 0.29 & 7 \\
\hline 2-Methylpher & 5.24 & 4.89 & 0.37 & 7 & CB 101 & 6.38 & 5.93 & 0.31 & 6 \\
\hline 3,6-Dimethylphenanthrene & $5.25^{\mathrm{a}}$ & 5.15 & 0.25 & 7 & CB 105 & 6.65 & 5.60 & 0.33 & 7 \\
\hline 2,6,9-Trimethylphenanthrene & $5.99^{\mathrm{a}}$ & 5.34 & 0.30 & 7 & CB 110 & 6.48 & 5.74 & 0.32 & 6 \\
\hline Dibenzothiophene & 4.38 & 4.04 & 0.31 & 7 & CB 112 & 6.45 & 5.59 & 0.31 & 7 \\
\hline Fluoranthene & 5.20 & 4.45 & 0.21 & 7 & CB 118 & 6.74 & 6.16 & 0.33 & 6 \\
\hline Pyrene & 5.00 & 4.49 & 0.17 & 7 & CB 128 & 6.74 & 6.10 & 0.42 & 7 \\
\hline 1-Methylfluoranthene & $5.48^{\mathrm{a}}$ & 5.01 & 0.19 & 7 & CB 132 & 6.58 & 5.79 & 0.29 & 7 \\
\hline 2,7-Dimethylpyrene & $6.03^{\mathrm{a}}$ & 6.30 & 0.27 & 7 & CB 137 & 6.83 & 6.10 & 0.35 & 6 \\
\hline Benzo[c]phenanthrene & $5.76^{*}$ & 5.38 & 0.18 & 7 & CB 138 & 6.83 & 6.52 & 0.31 & 6 \\
\hline Benz $[a]$ anthracene & 5.91 & 5.42 & 0.16 & 7 & CB 149 & 6.67 & 6.17 & 0.40 & 6 \\
\hline Chrysene & 5.86 & 5.23 & 0.15 & 7 & & 6.64 & 6.07 & 0.37 & 7 \\
\hline 2-Methylchrysene & $6.88^{+}$ & 6.15 & $0 . .20$ & 7 & CB 153 & 6.92 & 6.30 & 0.25 & 4 \\
\hline Benzo[b]fluoranthene & 5.78 & 6.33 & 0.09 & 7 & CB 156 & 7.18 & 7.26 & 0.34 & 5 \\
\hline Benzo[k]fluoranthene & 6.11 & 6.25 & 0.05 & 7 & CB 157 & 7.18 & 6.06 & 0.38 & 6 \\
\hline Benzo $[e]$ pyrene & 6.44 & 6.12 & 0.06 & 7 & CB 158 & 7.02 & 6.20 & 0.30 & 7 \\
\hline Benzo $[a]$ pyrene & 6.35 & 6.27 & 0.14 & 7 & CB 170 & 7.27 & 6.56 & 0.82 & 4 \\
\hline Perylene & 6.25 & 6.02 & 0.12 & 6 & CB 180 & 7.36 & 6.61 & 0.67 & 4 \\
\hline 7-Methylbenzo $[a]$ pyrene & $\mathrm{nf}$ & 6.97 & 0.18 & 6 & CB 183 & 7.2 & 6.67 & 0.42 & 4 \\
\hline Indeno $[1,2,3-c d]$ pyrene & 7.66 & 7.48 & 0.17 & 7 & CB 187 & 7.17 & 6.61 & 0.36 & 4 \\
\hline Benzo[ghi]perylene & 6.90 & 6.63 & 0.14 & 6 & & 7.24 & 6.45 & 0.40 & 4 \\
\hline Dibenz $[a, h]$ anthracene & 6.75 & 6.76 & 0.23 & 6 & CB 209 & 8.18 & 7.81 & 0.28 & 3 \\
\hline $\mathrm{D}_{12}$-Chrysene & $5.80^{\mathrm{b}}$ & 5.15 & 0.15 & 7 & & & & & \\
\hline $\mathrm{D}_{12}$-Benzo $[e]$ pyrene & $6.29^{\mathrm{c}}$ & 6.29 & 0.14 & 7 & & & & & \\
\hline
\end{tabular}

$3{ }^{1}$ C.I. $=$ Confidence interval of the estimate of the regression intercept $;{ }^{2} n=$ number of data points

4 making up plot; ${ }^{3} \log \mathrm{K}_{\text {ow }}$ of PAHs are from Sangster ${ }^{21},{ }^{\mathrm{a}}$ from ref $1,{ }^{\mathrm{b}}$ from ref $24,{ }^{\mathrm{c}}$ adopted

5 the value of Benzo[ $e]$ pyrene and those of CBs from Hawker and Connell ${ }^{27}, \mathrm{nf}=\mathrm{no} \log \mathrm{K}_{\mathrm{ow}}$ 
1 value found, ${ }^{+}$value adopted from (www.nature.nps.gov/hazardssafety/toxic/chrys c1.pdf), ${ }^{*}$

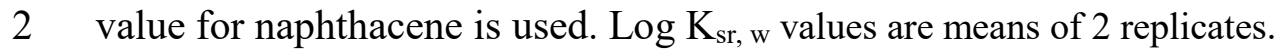

3 
1 Table 2: Log partition coefficients obtained by equilibration of 2 different silicone

2 rubbers

\begin{tabular}{|c|c|c|c|c|c|c|c|}
\hline Compounds & $\begin{array}{c}\text { ALTEC } \\
\text { Measured }^{1}\end{array}$ & VIZO $^{2}$ & $\begin{array}{c}\text { ALTEC } \\
\text { Estimated }^{3}\end{array}$ & Compound & $\begin{array}{c}\text { ALTEC } \\
\text { Measured }\end{array}$ & VIZO & $\begin{array}{c}\text { ALTEC } \\
\text { Estimated }\end{array}$ \\
\hline Phenanthrene & 4.18 & 3.89 & 4.06 & CB 28 & 4.79 & 5.22 & 5.36 \\
\hline Anthracene & 4.31 & 4.00 & 4.18 & CB 31 & 4.66 & 5.23 & 5.33 \\
\hline Fluoranthene & 4.45 & 4.38 & 4.59 & CB 44 & 5.21 & 5.56 & 5.71 \\
\hline Pyrene & 4.49 & 4.44 & 4.66 & CB 49 & 5.22 & 5.66 & 5.78 \\
\hline Benz[a]anthracene & 5.42 & 5.06 & 5.29 & CB 52 & 5.04 & 5.57 & 5.70 \\
\hline Chrysene & 5.23 & 4.97 & 5.20 & CB 101 & 5.93 & 6.03 & 6.13 \\
\hline Benzo[b]fluoranthene & 6.33 & 5.51 & 5.71 & CB 105 & 5.60 & 6.17 & 6.31 \\
\hline Benzo[ $k]$ fluoranthene & 6.25 & 5.51 & 5.71 & CB 118 & 6.16 & 6.20 & 6.30 \\
\hline Benzo[e]pyrene & 6.12 & 5.45 & 5.65 & CB 138 & 6.52 & 6.53 & 6.64 \\
\hline Benzo[a]pyrene & 6.27 & 5.52 & 5.71 & CB 153 & 6.30 & 6.45 & 6.54 \\
\hline Indeno[1,2,3- & & & & & & & \\
$c d]$ pyrene & 7.48 & 5.99 & 6.20 & CB 156 & 7.26 & 6.58 & 6.65 \\
\hline Benzo[ghi]perylene & 6.63 & 5.92 & 6.12 & CB 170 & 6.56 & 6.90 & 6.98 \\
\hline Dibenz[a, $h]$ anthracene & 6.76 & 6.04 & 6.26 & CB 180 & 6.61 & 6.84 & 6.90 \\
\hline D12-Chrysene & 5.15 & 4.91 & 5.14 & CB 187 & 6.61 & 6.77 & 6.84 \\
\hline
\end{tabular}

${ }^{1}$ Experimentally measured in this study; ${ }^{2}$ estimated using equation 4 and

$4 \quad \log K_{s r, w}$ values for the Vizo rubber from ref 17 
1 Table 3: Selected estimated $\log K_{s r, w}$ values from the $K_{s r, w}-\log K_{o w}$ regressions

\begin{tabular}{|c|c|c|c|}
\hline Compound & $\log \mathbf{K}_{\text {sr,w }}$ & S. E. $^{\mathbf{a}}$ & $\operatorname{log~K}_{\mathbf{o w}}$ \\
\hline \multicolumn{3}{|c|}{ PAHs } \\
\hline Acenaphthene & 3.78 & 0.09 & 3.92 \\
\hline Fluorene & 4.04 & 0.08 & 4.18 \\
\hline Phenanthrene & 4.36 & 0.07 & 4.52 \\
\hline Fluoranthene & 5.02 & 0.06 & 5.20 \\
\hline Chrysene & 5.66 & 0.07 & 5.86 \\
\hline Benzo[a]pyrene & 6.14 & 0.08 & 6.35 \\
\hline Benzo[g,h,i]perylene & 6.67 & 0.11 & 6.90 \\
\hline Dibenz[a,h]anthracene & 6.52 & 0.10 & 6.75 \\
\hline \multicolumn{3}{|c|}{ CBs } \\
\hline CB 31 & 4.81 & 0.08 & 5.67 \\
\hline CB 52 & 5.01 & 0.07 & 5.84 \\
\hline CB 101 & 5.64 & 0.05 & 6.38 \\
\hline CB 110 & 5.76 & 0.05 & 6.48 \\
\hline CB 118 & 6.07 & 0.05 & 6.74 \\
\hline CB 105 & 5.96 & 0.05 & 6.65 \\
\hline CB 183 & 6.60 & 0.07 & 7.20 \\
\hline CB 170 & 6.69 & 0.07 & 7.27 \\
\hline
\end{tabular}

$2{ }^{\text {a S.E. }}=$ Standard error of predicted value, $\log \mathrm{K}_{\mathrm{ow}}$ of PAHs are from Sangster ${ }^{21}$ and those of CBs

3 from Hawker and Connell ${ }^{27}$.

4

5 Table 4: Summary of linear regression analysis of $\log K_{s r, w}$ versus $\log K_{o w}$

\begin{tabular}{|c|c|c|c|c|c|c|}
\hline & $\log \mathrm{K}_{\text {ow }}$ & Slope & Intercept & $r^{2}$ & $s^{a}$ & $n^{b}$ \\
\hline \multirow{2}{*}{ PAHs } & Ref 21 & 0.97 & -0.01 & 0.94 & 0.29 & 24 \\
\cline { 2 - 7 } & Ref 26 & 1.13 & -0.79 & 0.94 & 0.38 & 17 \\
\hline \multirow{2}{*}{ CBs } & Ref 27 & 1.17 & -1.82 & 0.9 & 0.25 & 31 \\
\cline { 2 - 7 } & Ref 28 & 1.2 & -1.98 & 0.91 & 0.28 & 15 \\
\hline \multirow{2}{*}{$\begin{array}{c}\text { Pooled (PAHs \& } \\
\text { CBs) }\end{array}$} & $\begin{array}{c}\text { Ref 21 \& } \\
27\end{array}$ & 0.87 & 0.30 & 0.89 & 0.35 & 55 \\
\hline
\end{tabular}

${ }^{\mathrm{a}}$ standard deviation of the fit; ${ }^{\mathrm{b}}$ sample size 